\title{
To Access Remote Desktop using Windows Azure Cloud Computing
}

\author{
Saurabh Kapoor ${ }^{1}$, Prof. Ashok Verma ${ }^{2}$, Prof Ajay Lala \\ M.Tech Student, Gyan Ganga Institute of Technology and Sciences, Jabalpur, India ${ }^{1}$ \\ Associate Professor, Gyan Ganga Institute of Technology and Sciences, Jabalpur, India ${ }^{2,3}$
}

\begin{abstract}
This paper describe about an era of cloud computing platform in terms of Azure cloud platform and services. When choosing remote desktop control software in business environment following important decision to be made possible in cloud computing such as In house hosting, self hosting remote desktop for Software as a service tools. It defines the WCF RESTful services and Azure Appfabric Service Bus working together to allow thin client to access remote machine using a browser. It may consist of Azure service bus to communicate with application to share a target machine screen. The application running on a machine which helps us to allow the user to control and view the target machine desktop screen from browser.
\end{abstract}

Keywords: Cloud computing, WCF Service, REST Service,Azure Appfabric service bus.

\section{INTRODUCTION}

Cloud computing is an internet based services delivery result for developing and expanding in real world IT model in which services are hosted over the internet by a industry without added complexity. Using Azure cloud service provider. Cloud services give us a good platform data storage, backup and recovery have become infrastructure like hardware, servers and other respective more efficient and economical. It is also easier to build offers as a service enterprise.

The cloud services can be consumed by the enterprises for example channels like browser based user interface, mobile applications, smart client applications deployed in on-premises and other open channels like Windows Azure Marketplace, data market and application market.

Further, cloud services will interoperate with other programming platforms such as Java. Enterprises will significantly have an ownership and cost of application in cloud computing as on-premise deployment model. The cloud computing model also provides service provider for shared infrastructure across a number of enterprises. Typical billing models that are employed in the cloud paradigm are either subscription based or usage based or a combination of both. The billing models are also tied to strict SLA compliance by the service provider. It is more important to classify cloud deployment models, which are typically organized as Public Cloud, Hybrid Cloud, Private Cloud, and Community Cloud.

Windows Azure is Microsoft's cloud platform for growing collection of integrated services like computing, storage, data, networking, and App deployment that help us to express new way of developing and also to save money. But that's just scratching the surface. Windows Azure is the only cloud platform which is ranked by Gartner as an industry standard leading for both IaaS (infrastructure-as-a-service) and (PaaS) platform-as-aservice. Azure cloud platform is the combination of managed and unmanaged cloud services will help us to build, deploy, and manage our applications in real world for unmatched productivity.

Some cloud providers have option to choose either public cloud and datacenter but not Azure. It's also having an option for hybrid cloud solutions which gives us the best

\section{applications on cloud and on-premises also.}

Windows Azure can quickly scale up or down to match demand according to user's credentials i.e. user can only pay for what you use. It also gives us freedom for minute per minute billing and has commitment to match other competitor prices for popular infrastructure services like computing, storage and bandwidth which means you are always getting unbeatable price for performance.

\section{LITERATURE REVIEW}

The study of cloud computing and virtualization in embedded devices uses WCF for communication. It defines the demands an environment should fulfill in order to enable an efficient integration of embedded devices in a heterogeneous environment like the Web. In a service oriented environment, WCF services represent the basis for a middleware level in interaction between clients and embedded devices. Virtualization has been a hot topic in the enterprise space for quite some time, but has recently become an important technology for embedded systems as well or cloud based system and services. It is therefore important for embedded-systems developers to understand the power and limitations of virtualization in this space, in order to understand what technology is suitable for their products [1]. Virtualization is a term that refers to the abstraction of computer resources. Simply put, virtualization is a technique for hiding the physical characteristics of computing resources. The system or other applications will interact with those resources. The concept of virtualization is very broad and can be applied to devices, servers, operating systems, applications and even networks.

In Cloud environment like windows Azure as a platform as a service will provide us to deploy or create a services 
in azure cloud with highly security dependent. Now in application configuration file (app.config) in Virtualization is a term to communicate with other Microsoft visual Studio solution explorer values of applications which is hosted anywhere and also interacts issuerName and issuerSecret attributes in "sharedSecret" with each other without any additional resources.

Business will also extend with the help of cloud storage and various applications with effective cost and expenses. In the term of economy IT industry increases the new way of development and innovation in cloud computing with innovating things with keep up with the new needs of the business. Using cloud based platform developers are now starting to evaluate effective recourses to our business. To focuses these challenges in new era of cloud computing Microsoft gives us an effective platform as Windows Azure AppFabric service bus to build, deploy our application more easily with low cost.

\section{PROPOSED WORK}

The WCF Restful service acts as a light web server and the service bus will provide a remote access through browser to solve a problem of windows firewall and dynamic IP address. An application represents the two technologies. Firstly, An application which is running on a target machine which allows the user to see or view and also control the target machine screen from his/her browser.

Secondly, client browser will fetch an image of a remote machine screen which allow to operate various methods or control to operate various controls on the image which is viewed i.e. to implement this work we need in some extent like:1. A web server

2. Screen fetches mechanism

3. Control like clicking on Mouse and text writing to controls

The web server is implemented as self-contained RESTful WCF service which is able to receive HTTP requests and this request is generated by a desktop as a browser. The service exposes an endpoints to transfer text and image data to client. The endpoints have relay binding to support communication through Azure AppFabric Service Bus. This type of communication enables connection between browser and WCF service on target machine which is running in different networks with dynamic IP address. To implements this remote access and screen share application we first have an account on Windows Azure cloud platform as an AppFabric service and create a service namespace and install windows Azure development kit on target machine.

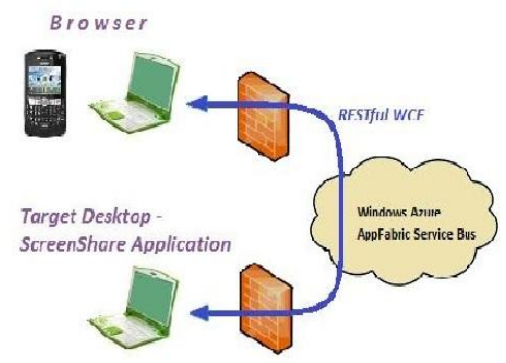
tag and placeholder like "service namespace" should be change with values of our Azure AppFabric Service Bus account. Application writes in console application

URL from which we accessed it is:

https://mysaurabh.servicebus.windows.net/ScreenShare/Re moteScreen

Output of ScreenShare application is shown in the following Figure:

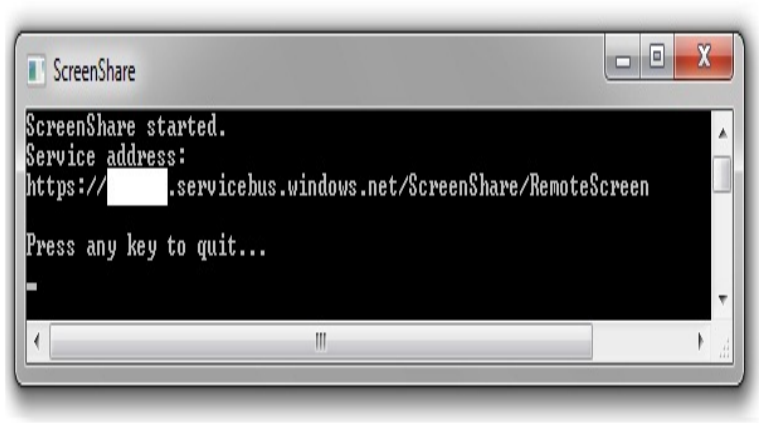

Fig.2 Console window Screen of service address

Now we can access the remote screen of the target machine from our browser using the URL which we have got from azure service bus. Our proposed work gives the functionality to client machine can get a fetched image of a target machine screen in browser and then the control target machine by clicking the left mouse and then the text which is inserted in browser and transmitted back to target machine. After clicking on left mouse click on the image which is shown in browser, the coordinates of the clicked point are transferred to target machine and then the application emulates the click at the same coordinates in the target machine screen. To transmit the text to the client should move the cursor to browser image of selected control. The text which is inserted will be transmitted to the target machine and placed into appropriate control. After click or text transfer, the image in client (browser) will be updated to reflect changes on target machine.

\section{RESULT}

In Azure cloud we create a service and when an application consume a service which is deployed in azure cloud. When an client application runs it generated a service uri in console screen and from the service uri we can access or share the remote screen images in browsers of the mobile devices with different magnification and resolution.

Mobile browsers functionality will work properly but some image size should be adjusted according to mobile browsers, and it is difficult to insert and manipulate some text. Actually HTML file and application itself should be tuned to serve at least the most popular browsers.

So far application was tested with Google chrome, Internet Explorer (IE), android, Windows Phone 8, iPhone, Firefox.

Fig.1 Remote Access Using Windows Azure Service 


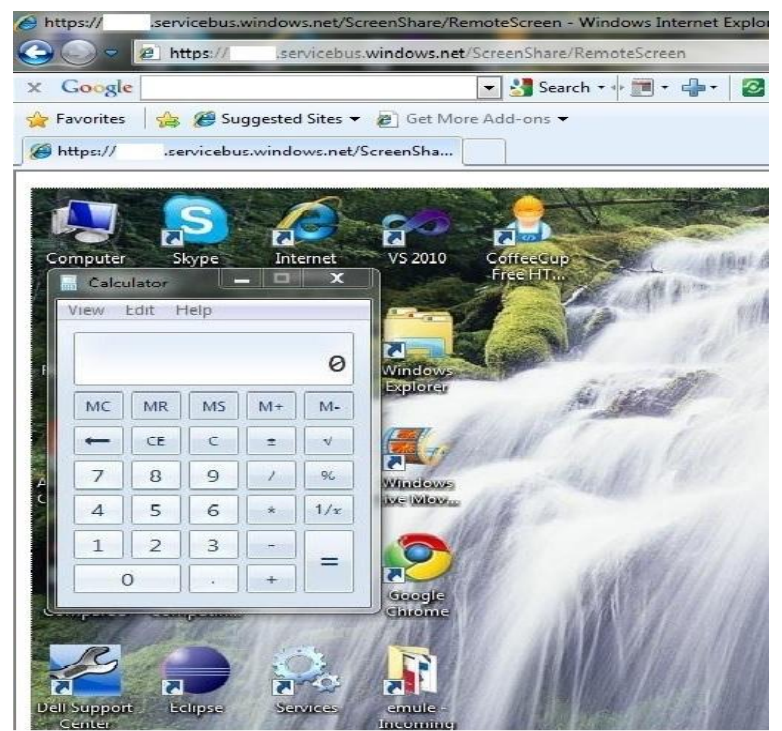

Fig 3.Screen access in Windows OS

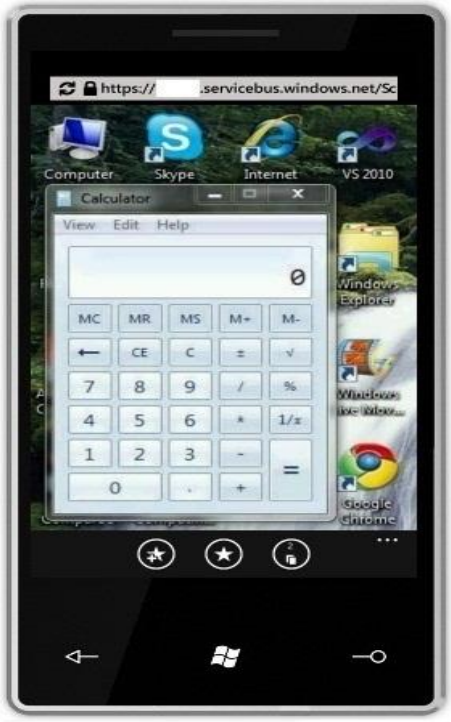

Fig 4.Screen access in Windows Phone

Screen images in different browsers of mobile devices were differ some magnification issues and also with some resolution resolution. The best results were achieved with Google chrome Internet Explorer, Firefox which shows the exact picture but sometime it act to fails to react in (RemoteScreen.html)file. Our work means to compete with sophisticated work done by using Azure cloud and service to communicate with each other. But the actual work done to describe the respective approach is that we any client as a browser without have any additional installation on machine used to operate desktop remotely. The ability of browser is very limited by using HTML and JavaScript.

\section{CONCLUSION}

The WCF Restful service and windows Azure AppFabric Service Bus application will approach and also allows user to access and control remote machine with any browser from any place. 